\title{
Modeling share returns - an empirical study on the Variance Gamma model
}

\author{
A. Rathgeber ${ }^{\mathrm{a}}$, J. Stadler ${ }^{\mathrm{a}}$, S. Stöckl ${ }^{\mathrm{b}, *}$ \\ ${ }^{a}$ FIM Research Center Finance \& Information Management, Department of Information Systems Engineering \& Financial \\ Management, University of Augsburg, Universitätsstrasse 12, 86159 Augsburg, Germany \\ ${ }^{b}$ Department of Economics, Chair for Corporate Finance (Visiting Professor), University of Constance, Box 141, \\ 78457 Constance, Germany
}

\begin{abstract}
Due to the fact that there has been only little research on some essential issues of the Variance Gamma (VG) process, we have recognized a gap in literature as to the performance of the various estimation methods for modeling empirical share returns. While some papers present only few estimated parameters for a very small, selected empirical database, Finlay and Seneta (2008) compare most of the possible estimation methods using simulated data. In contrast to Finlay and Seneta (2008) we utilize a broad, daily, and empirical data set consisting of the stocks of each company listed on the DOW JONES over the period from 1991 to 2011 . We also apply a regime switching model in order to identify normal and turbulent times within our data set and fit the VG process to the data in the respective period. We find out that the VG process parameters vary over time, and in accordance with the regime switching model, we recognize significantly increasing fitting rates which are due to the chosen periods.
\end{abstract}

Keywords: Variance Gamma Model; Parameter Estimation Methods; Regime Switching Model

JEL classification: G15

\section{Introduction}

Distribution assumptions and jump discontinuities play an important role in modeling share returns. Jumps can generate fat tails, and in doing so they can influence the excess skewness and kurtosis. Fama (1965) studies the distribution of daily returns on the Dow Jones Industrial Average Index (DOW JONES) over the period from 1957 to 1972. In his analysis, he includes the stocks of the thirty companies listed on the DOW JONES, rejects the normality assumption and suggests that non-normal distribution assumptions would probably fit more accurately. However, he is not able to find a Paretian stable distribution for

*Corresponding author: Tel: +49 (0)7531 88-3425 Fax: +49 (0)7531 88-3440

Email address: stefan.stoeckl@uni-konstanz.de (S. Stöckl) 
his non-normal distribution assumptions. According to Mandelbrot (1963) this means that the distributions have means but their variances are infinite and therefore, many commonly used statistical methods cannot be applied any longer. Based on these results Officer (1972) or Hsu et al. (1974) - amongst others demand better modeling approaches for non-stationary share returns as their attempts to estimate Paretian stable distributions (at least one finite mean and one variance) failed. Praetz (1972) was the first trying to improve share return modeling by means of an inverse gamma distribution which implies a rescaled Student t-distribution for returns. His results are superior to Fama's (1965) and are regarded as the basis for all future stochastic variance models.

Since Madan and Seneta (1987) took the results of Praetz (1972) and published the first symmetric version of the Variance Gamma process (VG process) with mean zero, there has been some progress in developing alternatives to the common Brownian Motion model for stock market returns, which had been developed by Osborne (1959). The Brownian Motion is an essential part of the Black-Scholes option pricing model devised by Black and Scholes (1973) although it does not include the third and fourth moment. This is in contrast to the VG process which contains all four moments. By integrating the jump diffusion model into the traditional Black-Scholes option pricing model, Merton (1976) was the first to succeed in taking the market's reactions on incoming information into account. Cox and Ross (1976), Jones (1984), Hull and White (1987), Heston (1993a) and Heston (1993b) - amongst others - follow the trend of modeling market fluctuations with Lévy processes or other stochastic processes with jumps. With regard to that they build on a diffusion concept which has a martingale component with sample paths being continuous functions of calendar time. Madan and Seneta (1990) extend the Black-Scholes model by applying the VG process within the pricing framework to the Variance Gamma option pricing model. Madan et al. (1998) conclude that Variance Gamma option pricing reduces the pricing bias - in contrast to the Black-Scholes model - as the VG process covers the excess kurtosis, which is a result of jumps. Daal and Madan (2005) use this new idea for an empirical examination of the Variance Gamma option pricing model, the traditional Black-Scholes model and Merton's (1976) jump diffusion model for foreign currency options. They reaffirm Madan and Seneta's (1990) findings that the Variance Gamma option pricing model performs better than the others do. In contrast to the two-parametric Brownian Motion the VG process mentioned above is a four-parametric stochastic process. Therefore, these two methods used for stock market modeling differ widely as the VG process captures the skewness and kurtosis in addition to the mean and standard deviation. The VG process considers both, the symmetric increase in the left and right tail probabilities of the return distribution (kurtosis) and the asymmetry of the left and right tails of the return density (skewness). These properties allow for a more accurate representation of stock returns. The VG process parameters can be obtained by the application of several methods, such as the simplified method of moments, the method of moments, the maximum likelihood estimation, the empirical characteristic function, the Bayesian inference and Markov chain Monte Carlo method, or the minimum $\chi^{2}$ method. For an overview see Madan and Seneta (1987), Madan and Seneta (1990), Seneta (2004) and particularly Finlay and Seneta (2008).

However, there has been only little research on some essential issues of the VG process, so far. We have recognized a gap in literature as to the performance of the various estimation methods for modeling empirical share returns. While some papers present only few estimated parameters for a very small, selected empirical database, Finlay and Seneta (2008) compare most of the possible estimation methods using simulated data. In contrast to Finlay and Seneta (2008), we utilize a broad, daily, and empirical data set consisting of the stocks of each of the companies listed on the DOW JONES over the period from 1991 to 2011. Additionally, the calibration quality as well as the parameters' range of the VG process are 
dependent on time. This means that parameters vary in different market phases. As market participants are exposed to varying situations, the selection of the correctly fitted model is an essential element of their work. This leads us to apply a regime switching model in order to identify normal and turbulent regimes within our data set and to fit the VG process to the data in the respective period. This approach has two major advantages. First, it results in a more accurate parameter estimation, which avoids overor underestimation of the real VG process. Second, the fitting rate - the fact that the returns follow a VG process - increases significantly. Thus, the use of the regime switching model adds new knowledge to the framework of the VG process.

The remainder of this paper is structured as follows. The next section provides an overview of the theoretical background of the VG process itself, the several estimation methods for the VG process parameters, and our hypotheses. Section 3 introduces the research design including the data set and the methodology of differentiating between normal and turbulent times in financial markets. Section 4 presents the results of the VG process parameter estimation. Subsequent to the discussion of the results in section 5, section 6 concludes the paper.

\section{Theoretical Background}

This section presents the framework for modeling risky assets by means of a VG process and provides a brief overview of the estimation methods for the VG process parameters. We define the price of a risky asset $S_{t}$ at point in time $t(t \geq 0)$ with the following traditional model

$$
S_{t}=S_{0} e^{r_{t}}
$$

where $r_{t}=\ln \frac{S_{t}}{S_{0}}$ is the compounded $\log$ return from $t=0$ to $t . r_{t}$ follows the stochastic process $r_{t}=c t+\theta T_{t}+\sigma W\left(T_{t}\right)$, where $c, \theta$ and $\sigma(>0)$ are real constants as defined by Seneta (2004). $W(\cdot)$ represents the traditional Brownian Motion. Therefore, this approach is also known as the VG process as a modified Brownian Motion. Besides, Schoutens (2003) and Cont and Tankov (2004) describe the VG process as the difference between two independent gamma processes. Luciano and Schoutens (2006) take these results and define a Lévy Triplet by means of the difference between two gamma processes. Seneta and Tjetjep (2006) model the VG process as a normal-variance-mean-mixture-model. Seneta (2004) models the market activity time $\left(T_{t}\right)_{t \geq 0}$ as a positive, monotonically non-decreasing random process with stationary increments $\tau_{t}=T_{t}-T_{t-1}(t \geq 1)$ and $T_{0}=0$ (almost surely). It is assumed that the expected duration of an increment is $E\left(\tau_{t}\right)=1 \forall t \geq 0$. This simplification allows normalizing the expected economically relevant measure to 1 . Seneta (2004), Finlay and Seneta (2008) - amongst others - use the corresponding increments $X_{t}$ of $S_{t}$ instead of the compounded return $r_{t}$ for fitting the VG process. This means that $X_{t}=c+\theta\left(T_{t}-T_{t-1}\right)+\sigma\left(W\left(T_{t}\right)-W\left(T_{t-1}\right)\right)$ and from the distribution properties of $W(\cdot), \sigma\left(W\left(T_{t}\right)-W\left(T_{t-1}\right)\right) \stackrel{\mathrm{D}}{=} \sigma \sqrt{T_{t}-T_{t-1}} W(1)$, the relevant formula for $X_{t}$ can be derived

$$
X_{t}=c+\theta \tau_{t}+\sigma \sqrt{\tau_{t}} W(1)
$$


Both, Seneta (2004) and Finlay and Seneta (2006) model $\tau_{t}$ with a gamma distribution $\gamma\left(\frac{1}{\nu}, \frac{1}{\nu}\right)^{1}$ with $\nu>0$ and a gamma function $\Gamma\left(\frac{1}{\nu}\right)$ embedded in the gamma probability density function (PDF)

$$
f_{\tau}(w)= \begin{cases}\frac{1}{\nu^{\frac{1}{\nu}}} \frac{w^{\frac{1}{\nu}-1} e^{\frac{-w}{\nu}}}{\Gamma\left(\frac{1}{\nu}\right)}, & w, \nu>0 \\ 0, & \text { else }\end{cases}
$$

which fulfills the requirements that $E\left(\tau_{t}\right)=1$ and $\operatorname{Var}\left(\tau_{t}\right)=\nu$. Before Madan and Seneta (1990) introduced the VG process in 1990, there had not existed a closed form for the PDF of the VG process. They only identified a closed form for the characteristic function. By means of a Bessel function $K$ it is possible to close this research gap (see Cont and Tankov (2004)) and to define $f_{X}(x)$ as the PDF of the VG process

$$
f_{X}(x)=\frac{2}{\sigma \sqrt{2 \pi} \nu^{\frac{1}{\nu}} \Gamma\left(\frac{1}{\nu}\right)} e^{\theta \frac{x-c}{\sigma^{2}}}\left(\frac{|x-c|}{\sqrt{\frac{2 \sigma^{2}}{\nu}+\theta^{2}}}\right)^{\frac{1}{\nu}-\frac{1}{2}} K_{\frac{1}{\nu}-\frac{1}{2}}\left(\frac{|x-c| \sqrt{\frac{2 \sigma^{2}}{\nu}+\theta^{2}}}{\sigma^{2}}\right),
$$

with $\theta, \sigma, \nu$ and $c$ representing the VG process parameters, according to Seneta (2004). As suggested by both, Madan and Seneta (1990) and Cont and Tankov (2004), we apply the Bessel function of the second kind. Nevertheless, there is another concept developed by Seneta (2004), where $K$ is modeled as the Bessel function of the third kind. The characteristic function $\phi_{X}(x)=E\left(e^{i u x}\right)$ for the VG process can be generated by means of the PDF (see formula 4) and Madan et al. (1998) define it as

$$
\phi_{X}(u)=e^{i c u}\left(1-i \theta \nu u+\frac{\sigma^{2} \nu u^{2}}{2}\right)^{-\frac{1}{\nu}},
$$

with $-\infty<u<\infty$ and $i=\sqrt{-1}$. On the basis of these properties the four moments of the VG process can be calculated. By using the moment generating function $m_{\tau}(x)=E\left(e^{u x}\right)$ for the time increments $\tau_{t}, m_{\tau}(u)=(1-\nu u)^{-\frac{1}{\nu}}$, the $i$-th moment $E\left[\left(\tau_{t}-0\right)^{i}\right]$, with $i=1, \ldots, 4$, about zero, the $i$-th central moment $M_{i}=E\left[\left(\tau_{t}-1\right)^{i}\right]$, with $i=1, \ldots, 4$, we get $E\left[\left(X_{t}-E\left(X_{t}\right)\right)^{2}\right]=c+\theta$, $E\left[\left(X_{t}-E\left(X_{t}\right)\right)^{2}\right]=\sigma^{2}+\theta^{2} M_{2}, E\left[\left(X_{t}-E\left(X_{t}\right)\right)^{3}\right]=3 \theta \sigma^{2} M_{2}+\theta^{3}$ and $E\left[\left(X_{t}-E\left(X_{t}\right)\right)^{4}\right]=$ $3 \sigma^{4}\left(1+M_{2}\right)+6 \sigma^{2} \theta^{2}\left(M_{2}+M_{3}\right)+\theta M_{4}$. Finally, we present the four moments $\tilde{M}_{i}$, with $i=1, \ldots, 4$, of the VG process mean $(\mu)$, variance $\left(\sigma^{2}\right)$, skewness $(\beta)$ and kurtosis $(\kappa)$ depending on the parameter set $\eta=(\sigma, \theta, \nu, c)$

$$
\begin{aligned}
& \tilde{M}_{1}:=\hat{\mu}=c+\theta \\
& \tilde{M}_{2}:=\hat{\sigma^{2}}=\sigma^{2}+\theta^{2} \nu \\
& \tilde{M}_{3}:=\hat{\beta}=\frac{2 \theta^{3} \nu^{2}+3 \sigma^{2} \theta \nu}{\left(\sigma^{2}+\theta^{2} \nu\right)^{\frac{3}{2}}} \text { and } \\
& \tilde{M}_{4}:=\hat{\kappa}=3+\frac{3 \sigma^{4} \nu+12 \sigma^{2} \theta^{2} \nu^{2}+6 \theta^{4} \nu^{2}}{\left(\sigma^{2}+\theta^{2} \nu\right)^{2}} .
\end{aligned}
$$

\footnotetext{
${ }^{1}$ In general, a $\gamma(b, p)$ distribution has $f(x)=\frac{b^{p}}{\Gamma(p)} x^{p-1} e^{-b x} \forall b, p, x>0$ as PDF.
} 
Seneta and Tjetjep (2006) calculate some upper and lower bounds for the skewness and the kurtosis. All the bounds depend on the subordinated VG process with its parameter $\nu$. The skewness is embedded in the range $-3 \sqrt{\nu}<\beta<3 \sqrt{\nu}$ and the kurtosis is embedded in the range $3 \nu+3<\kappa<6 \nu+3$. For a more detailed derivation of the respective formulas see, for example, Madan and Seneta (1987), Madan and Seneta (1990) and Seneta (2004).

For fitting the PDF of the VG process and the normalized moments mentioned above, the related literature suggests several approaches. In the subsequent paragraphs we provide a short overview of the six different VG process estimation methods.

$\underline{\text { Simplified method of moments }}$

The simplified method of moments (SMOM) approach is the simplest way of determining the parameter set $\eta$ of a VG process. Seneta (2004) and Finlay and Seneta (2006) assume that we face a symmetric case of the empirical distribution of the $\log$ returns and we can approximate $\theta \approx 0$. This restriction implies $\theta^{2}=\theta^{3}=\theta^{4}=0$ and by means of the empirical moments $M_{i}^{E}$, with $i=1, \ldots, 4$, we get the following four estimators for

$$
\sigma^{*}=\sqrt{M_{2}^{E}}, \quad \nu^{*}=\frac{M_{4}^{E}}{3}-1, \quad \theta^{*}=\frac{M_{3}^{E} \sigma^{*}}{3 \nu *} \text { and } \quad c^{*}=M_{1}^{E}-\theta^{*} .
$$

The results of the SMOM will be used as the starting parameters for the following optimization methods.

\section{Method of Moments}

The method of moments (MOM) is a numerical algorithm trying to identify a parameter set for the moments which is very close to the empirical one. As Finlay and Seneta (2008) suggest, we use the least-square method and minimize the relative deviation between the $i$-th sample moment $M_{i}^{E}$ calculated from the empirical data set and the $i$-th moment $\tilde{M}_{i}$, with $i=1, \ldots, 4$ (see formulas 6 - 9), derived from the parameter set. From this framework we get the optimization model

$$
\left(\sigma^{*}, \theta^{*}, \nu^{*}, c^{*}\right)_{M O M}=\underset{\sigma, \theta, \nu, c}{\operatorname{argmin}} \sum_{i=1}^{4}\left(\frac{M_{i}^{E}-\tilde{M}_{i}(\sigma, \theta, \nu, c)}{M_{i}^{E}}\right)^{2},
$$

with $\sigma^{*}>0$ and $\nu^{*}>0$. Seneta and Tjetjep (2006) modify the optimization to

$$
\left(\sigma^{*}, \theta^{*}, \nu^{*}\right)_{M O M}=\underset{\sigma, \theta, \nu}{\operatorname{argmin}} \sum_{i=2}^{4}\left(\frac{M_{i}^{E}-\tilde{M}_{i}(\sigma, \theta, \nu)}{M_{i}^{E}}\right)^{2}
$$

and $c^{*}=M_{1}^{E}-\theta^{*}$, as the parameter $c$, only has an effect on $\tilde{M}_{1}$.

\section{$\underline{\text { Maximum Likelihood Estimation }}$}

The maximum likelihood estimation (MLE) is a method for evaluating the density of an $N$-dimensional 
random process. Based on the ideas of Efron (1982) and Aldrich (1997), as suggested by Seneta (2004), we use the MLE for fitting the VG process to the parameter set $\eta=(\sigma, \theta, \nu, c)$ at $X_{i}$, with $X=X_{1}, \ldots, X_{N}$ representing the random log-return process. Finally, we optimize

$$
f_{X}\left(x \mid \sigma^{*}, \theta^{*}, \nu^{*}, c^{*}\right)_{M L E}=\underset{\sigma, \theta, \nu, c}{\operatorname{argmax}} \prod_{i=1}^{N} f_{X}(x \mid \sigma, \theta, \nu, c),
$$

where $\prod_{i=1}^{N} f_{X}(x \mid \sigma, \theta, \nu, c)$ is defined as the so-called likelihood function $\mathcal{L}(\eta)$. As we face i.i.d samples of $X$, Aldrich (1997) concludes that we can use the practical log-likelihood function $\mathcal{L} \mathcal{L}(\eta)=$ $\ln (\mathcal{L}(\eta))$ for the maximization

$$
f_{X}\left(x \mid \sigma^{*}, \theta^{*}, \nu^{*}, c^{*}\right)_{M L E}=\underset{\sigma, \theta, \nu, c}{\operatorname{argmax}} \sum_{i=1}^{N} \ln \left(f_{X}(x \mid \sigma, \theta, \nu, c)\right) .
$$

With respect to the PDF of the VG process (see formula 4) and the parameter $\nu$ used for defining the Bessel function of the second kind, we have to use a numerical approach as the partial derivation is not known. Due to the fact that the surface of the vicinity of the moments is very likely to be flat, previous optimization approaches are unsuccessful. Therefore Seneta (2004) modifies the log-likelihood approach as follows. First, he suggests that all the observations of the random VG process are adjusted by the first empirical moment $M_{1}^{E}$ in order to get a VG process of the three parameters $\sigma, \theta$ and $\nu$ with the PDF

$$
f_{X}(z)=\frac{2}{\sigma \sqrt{2 \pi} \nu^{\frac{1}{\nu}} \Gamma\left(\frac{1}{\nu}\right)} e^{\theta \frac{z-\theta}{\sigma^{2}}}\left(\frac{|z-\theta|}{\sqrt{\frac{2 \sigma^{2}}{\nu}+\theta^{2}}}\right)^{\frac{1}{\nu}-\frac{1}{2}} K_{\frac{1}{\nu}-\frac{1}{2}}\left(\frac{|z-\theta| \sqrt{\frac{2 \sigma^{2}}{\nu}+\theta^{2}}}{\sigma^{2}}\right)
$$

with $z=X_{t}-M_{1}^{E}$. After this optimization, $c^{*}$ has to fulfill the requirement that $c^{*}+\theta^{*}=\frac{1}{N} \sum_{i=1}^{N} X_{i}$.

\section{Empirical characteristic function}

The empirical characteristic function (ECF) method tries to match the characteristic function derived from the VG process model with the empirical characteristic function obtained from empirical data. Seneta (2004) states that this approach has some advantages compared to the MLE as this method can sometimes cause maximization problems as mentioned in the previous paragraph. In accordance with Yu (2004), we first calculate an estimator for the empirical characteristic function $\Phi_{E C F}(\omega)$ using the $N$ observed log-returns $X=\left(X_{1}, \ldots, X_{N}\right)$

$$
\Phi_{E C F}(\omega)=\frac{1}{N} \sum_{j=1}^{N} e^{i \omega X_{j}}
$$

where $i=\sqrt{-1}$ and $\omega$ are the evaluation points. Then we take the characteristic function of the VG process $\Phi_{X, \eta}(\omega)$ (for a detailed overview see function 5) and set up the optimization problem

$$
\eta^{*}=\underset{\eta}{\operatorname{argmin}} \int_{-\infty}^{+\infty}\left|\Phi_{X, \eta}(\omega)-\Phi_{E C F}(\omega)\right|^{2} e^{-\omega^{2}} d \omega
$$


for estimating the parameter set $\eta^{*}$, this is in accordance with $\mathrm{Yu}(2004)$.

Bayesian inference and Markov chain Monte Carlo

Finlay and Seneta (2008) suggest the so-called Bayesian inference and Markov chain Monte Carlo (BI) method. Roberts (1965) already recommends the approach to look at the posterior distribution of the parameters of a data distribution which is usually the major objective of a Bayesian statistical analysis. This means that the posterior probability is the probability of the parameter set $\eta$ which is based on the evidence of $X: f_{X}(\eta \mid x)$, where $X=\left(X_{1}, \ldots, X_{N}\right)$ represents the $N$ observed log returns. Furthermore, the likelihood function $\mathcal{L}(\eta)$ describes the probability of the evidence given $f_{X}(x \mid \eta)$. Finally, assuming a prior probability $f_{X}(\eta)$, we can use the Bayes theorem and calculate the posterior probability $f_{X}(\eta \mid x)$ as

$$
f_{X}(\eta \mid x)=\frac{\mathcal{L}(\eta) f_{X}(\eta)}{\int \mathcal{L}(\eta) f_{X}(\eta) d \eta}
$$

On the basis of function 18 the mean of the posterior probability $\eta^{*}$ is defined as

$$
\eta^{*}=\mu\left(f_{X}(\eta \mid x)\right) .
$$

As already mentioned in the context of equations 13 and 14, there are some difficulties in handling the Bessel function. In this case it is hardly possible to find a solution to the integral $\int \mathcal{L}(\eta) f_{X}(\eta) d \eta$. Roberts and Rosenthal (2004) propose a Markov chain Monte Carlo (MCMC) simulation as a possible solution, because Markov's solution is similar to the original problem. In accordance with Finlay and Seneta (2008) we use WinBUGS for the MCMC simulation as it was designed for the Bayesian estimation of parameters. We run the MCMC simulation as described by Finlay and Seneta (2008) in detail.

$\chi^{2}$ method

Another estimation approach is the minimum $\chi^{2}\left(\chi^{2}\right)$ method. Berkson (1980) defines the chi-square function as a function of the observed frequencies and their expectations or estimates of their expectations which are asymptotically distributed in the tabular chi-square distribution. Finlay and Seneta (2008) confirm that this method is a very good approximation of the empirical PDF by means of the estimated PDF. While Berkson (1980) mentions five different versions of the $\chi^{2}$ method we use the approach proposed by Finlay and Seneta (2008).

The basis of this method are $N$ observed $\log$ returns $X=\left(X_{1}, \ldots, X_{N}\right)$ and the determination of $I$ intervals with a vector $B:=\left(B_{i}\right)_{i=0, \ldots, I}$ as right borders of the intervals. The number of the expected observations $\tilde{O}_{i}(\eta)$ in any interval is dependent on the parameter set $\eta$ and can be approximated with

$$
\tilde{O}_{i}(\eta)=N \int_{B_{i-1}}^{B_{i}} f_{X, \eta}(x) d x
$$

where $f_{X, \eta}(x)$ is the density function of the VG process (see formula 4). Finlay and Seneta (2008) suggest that the log returns are divided into $1 \%$ sample quantile bands. This means that $O_{i}$ is a constant over all intervals and has a value of $\frac{N}{100}$. We set the integration bounds to $B_{0}=-10$ and $B_{I}=$ 
10 , because a numerical integration to infinity is not possible due to the properties of $f_{X, \eta}(x)$. The optimization problem

$$
\eta^{*}=\underset{\eta}{\operatorname{argmin}} \sum_{i=1}^{M} \frac{\left(O_{i}-\tilde{O}_{i}(\eta)\right)^{2}}{\tilde{O}_{i}(\eta)}
$$

minimizes the relative difference between the observed and expected numbers of log returns in the determined intervals and in this way identifies the optimal parameter set $\eta^{*}$.

For testing the quality of the parameter estimation methods we use the Kolomogorov-Smirnov (KS) test as well as the $\chi^{2}$ test. An overview of these two distribution tests can be found in appendix A1.

Having described the theoretical background, we can develop some hypotheses. All hypotheses derive from the characteristic behavior of the stock markets and the estimation methods of the VG process parameters. So far, Finlay and Seneta (2008) have been the only authors who conducted a broad comparison of the estimation methods of the VG process. Their findings are mainly based on a simulated VG process data set and could be summarized, that in most instances, the MLE method is the superior method and that the $\chi^{2}$ estimation is - on average - the next best method. In accordance with Madan and Seneta (1987), Madan and Seneta (1990) and Daal and Madan (2005), who use only one or two estimation methods, there is no evidence for a large empirical data set. For the purpose of closing this gap, we assimilate the properties of the estimation methods and the typical features of empirical asset returns. The SMOM can be obtained from the empirical data set more or less directly. The empirical log returns often include outliers due to extreme events at the stock markets. These events affect the kurtosis directly and thus, produce some bias. Therefore, the minimization problem of the MOM leads to an overestimation of the parameter $\nu$ and thus, to a higher market activity as to the gamma function itself. The ECF method is closely related to the MOM. We have derived the four moments of the VG process from the moment generating function, which is similar to the characteristic function. For that reason, the ECF method is frequently confronted with the same overestimation of $\nu$. The BI method is independent of a particular optimization approach and relies on a simulation. As it is true for all simulations, the results are affected by the assumptions made within the simulation framework, such as the prior distribution of the parameter set $\eta$ or the intervals for the parameter set $\eta$. Nevertheless, this approach should provide more stable and better results compared to the SMOM, MOM and ECF method. The MLE and the $\chi^{2}$ methods are two approaches, which directly use the PDF for fitting the VG process. While the MLE method modifies the PDF to a three-parametric PDF for numerical reasons, the $\chi^{2}$ method utilizes the original PDF. The use of the PDF enables a close fitting between the empirical PDF and the PDF of the VG process. Particularly, the problem of extreme events can be captured more easily as the $\chi^{2}$ method employs intervals being able to handle these effects. Therefore, it can be stated that the $\chi^{2}$ method could be superior to that of the MLE. We build the first hypothesis (H1) on these fundamental aspects:

\section{H1: The $\chi^{2}$ method provides the best approximation of the VG process for an} empirical data set.

According to Hamilton (1989), Jeanne and Masson (2000) or Cerra and Saxena (2003) - amongst others - 
the stock markets' behavior can change from normal (= non-volatile) times to turbulent (=volatile) times over a longer period as a result of structural breaks. This market attitude can also affect the VG process' fitting procedure and the quality of the estimated parameter set $\eta$. Therefore, the fact whether the VG process is fitted to a data set taken from a normal time, or a turbulent time, or a mixture of both can be important. For example, the use of a badly selected data set can imply that the variance influencing parameter $\sigma$ under- or overestimates the real VG process or that the asymmetry component $\theta$ is biased. With this knowledge, we apply a regime switching model (for an overview of this model see Hamilton (1989) or Hamilton (2005)) for the purpose of identifying normal and turbulent times at the stock markets and calibrate the VG process using this selected database. Therefore, we formulate the next hypothesis (H2):

H2: If a stock faces different market phases, the parameter set $\eta$ of the VG process must vary over time.

Previous literature on the VG process' fitting models - e.g. Seneta (2004), Seneta and Tjetjep (2006) and Finlay and Seneta (2008) - use simulated data or only an empirical data set taken from a short period of time for the whole sample. Due to insufficient empirical testing of the above mentioned six fitting methods for large data sets, we use the DOW JONES as well as all index stocks over the period from 1991 to 2011. In accordance with hypothesis $\mathrm{H} 2$ and the assumption that the inclusion of a regime switching model contributes to a parameter improvement we state our last hypothesis (H3):

H3: The inclusion of a regime switching model in a VG process' framework increases the fitting rate of each parameter estimation method.

We can state that $\mathrm{H} 1$ provides a hint for the results of the other two hypotheses. Furthermore, $\mathrm{H} 2$ is closely connected to $\mathrm{H} 3$. Therefore, the tests of these hypotheses will also be joined in section 4 . 


\section{Research Design}

\subsection{Data}

In general, we must distinguish between the data set needed for fitting the VG process and the data set needed for the regime switching model. For analyzing the VG process we use daily returns of DOW JONES stocks and of the index itself over the period from 01.01.1991 to 31.12.2011. The stocks represent the actual composition of the DOW JONES at the end of the year 2011. With this framework we calibrate the VG process for 29 stocks and the index itself. We only exclude Kraft Foods Inc. from our research, because they went public only in June 2001. Therefore only limited daily returns are available.

Furthermore, we calibrate the regime switching model with weekly closing prices of the DOW JONES. For back-testing the results of this model we take the yields of United States of America (US) Treasuries with a maturity of 6 months (m), 12 months, 5 years (y) and 10 years as well as the closing prices of gold. Using the daily data set we conduct a correlation analysis based on the theoretical background of the safe haven theory.

Our data are provided by Thomson Reuters Datastream. Table 1 summarizes the data set and table 12 in appendix A1 provides some descriptive information - such as mean and standard deviation - about the data set. Our data set includes the different phases of the past 21 years. It covers bullish markets

\begin{tabular}{lccc}
\hline \hline Underlying & Timeframe & Interval & Observations \\
\hline Dow Jones Industrial Average Index & 01.01 .1991 to 31.12 .2011 & daily & 5298 \\
Dow Jones Industrial Average Index & 01.01 .1991 to 31.12 .2011 & weekly & 1094 \\
All Dow Jones Industrial Average Index stocks & 01.01 .1991 to 31.12 .2011 & daily & 5298 \\
US Treasury 6 Months Middle Rate & 01.01 .1991 to 31.12 .2011 & daily & 5298 \\
US Treasury 12 Months Middle Rate & 01.01 .1991 to 31.12 .2011 & daily & 5298 \\
US Treasury 5 Years Middle & 01.01 .1991 to 31.12 .2011 & daily & 5298 \\
US Treasury10 Years Middle & 01.01 .1991 to 31.12.2011 & daily & 5298 \\
gold & 01.01 .1991 to 31.12 .2011 & daily & 5298 \\
\hline
\end{tabular}

Table 1: Empirical data overview

which could be observed in the periods from 1991 to 1998 and from 2003 to 2007, the dot-com hype and the crisis subsequent to it, the events of 9/11, the financial crisis as well as the effects of the European sovereign debt crisis on US stocks. This broad range of data allows us to provide a profound analysis of the VG process in the next sections.

\subsection{Sector classification}

In some cases, it can be useful to group shares into sectors in order to identify common behavior and trends in different market phases. We use the North American Industry Classification System (NAICS) for classifying the companies listed on the DOW JONES into sectors. As to that, the classification system of the NAICS employs a six-digit code. While the first five digits are of general nature, the last digit indicates the national industries. The first two digits indicate the largest business sector, the third digit the sub-sector, the fourth digit the industry group, and the fifth digit the particular industry. For a detailed overview of the NAICS, see http://www.census.gov/cgi-bin/sssd/naics/naicsrch. For instance, it often suffices to rely on the first two digits. In some cases, however, such as in the manufacturing sector, 
which a lot of companies listed on the DOW JONES operate in, the third digit has to be employed. We identify the following six sectors, which sometimes cover more than one industry. For an overview see table 2 .

\begin{tabular}{|c|c|}
\hline Sector & Coverage \\
\hline information \& entertainment & $\begin{array}{l}\text { information sector } \\
\text { entertainment sector }\end{array}$ \\
\hline finance \& insurance & $\begin{array}{l}\text { financial sector } \\
\text { insurance sector }\end{array}$ \\
\hline engineering & $\begin{array}{l}\text { transportation equipment manufacturing } \\
\text { machinery manufacturing } \\
\text { primary metal manufacturing }\end{array}$ \\
\hline trade \& food & $\begin{array}{l}\text { retail trade } \\
\text { wholesale } \\
\text { food industry }\end{array}$ \\
\hline chemistry \& oil & $\begin{array}{l}\text { chemical manufacturing } \\
\text { petroleum and coal products manufacturing }\end{array}$ \\
\hline electrical \& component & $\begin{array}{l}\text { electrical sector } \\
\text { electrical component sector }\end{array}$ \\
\hline
\end{tabular}

Table 2: Sectors covered by the DOW JONES according to NAICS

This structure allows us to assign the DOW JONES stocks to sectors as presented in table 3 .

\begin{tabular}{llllll}
\hline \hline $\begin{array}{l}\text { information } \\
\& \text { entertainment }\end{array}$ & $\begin{array}{l}\text { finance } \\
\& \text { insurance }\end{array}$ & engineering & $\begin{array}{l}\text { trade } \\
\& \text { food }\end{array}$ & $\begin{array}{l}\text { chemistry } \\
\& \text { oil }\end{array}$ & $\begin{array}{l}\text { electrical } \\
\text { \& component }\end{array}$ \\
\hline ATT & AMEX & ALCOA & COCA COLA & CHEVRON & 3M \\
MICROSOFT & BOA & CAT & HOME DEPOT & DU PONT & CISCO \\
VERIZON & JP MORGAN & UTC & MCDONALDS & EXXON & GE \\
WALT DISNEY & TRAVELERS & & WAL MART & MERCK & HP \\
& & & JNJ & PFIZER & INTEL \\
& & & PG & & IBM \\
\hline
\end{tabular}

Table 3: Classification of DOW JONES into sectors according to NAICS

\subsection{Identification of normal and turbulent timeframes}

For identifying normal and turbulent timeframes in our economic time series we use a regime switching model. The change from a normal to a turbulent timeframe is often connected with a break in the time series caused by a financial crisis or by political changes (for an overview see Hamilton (1988), Jeanne and Masson (2000) and Cerra and Saxena (2003)). For an overview of the main idea of a regime switching model see Hamilton (1988), Hamilton (1989) or Hamilton (2005). We follow Hamilton (1989) 
and Hamilton (1994) by using the maximum likelihood estimation method ${ }^{2}$ for estimating the transition matrix $P^{*}$, which indicates the time series switches from state $i$ to state $j$. Our objective is to determine periods when the DOW JONES was in a normal market period or in a turbulent period respectively (resp.). For calibrating the regime switching model we use a database of weekly closing prices from January 1991 to December 2011. We prefer the weekly database to the daily database as the results are more stable. Figure 1 shows the development of the DOW JONES as well as the smoothed probability that the share index is in a turbulent regime. By means of these smoothed probabilities, we divide

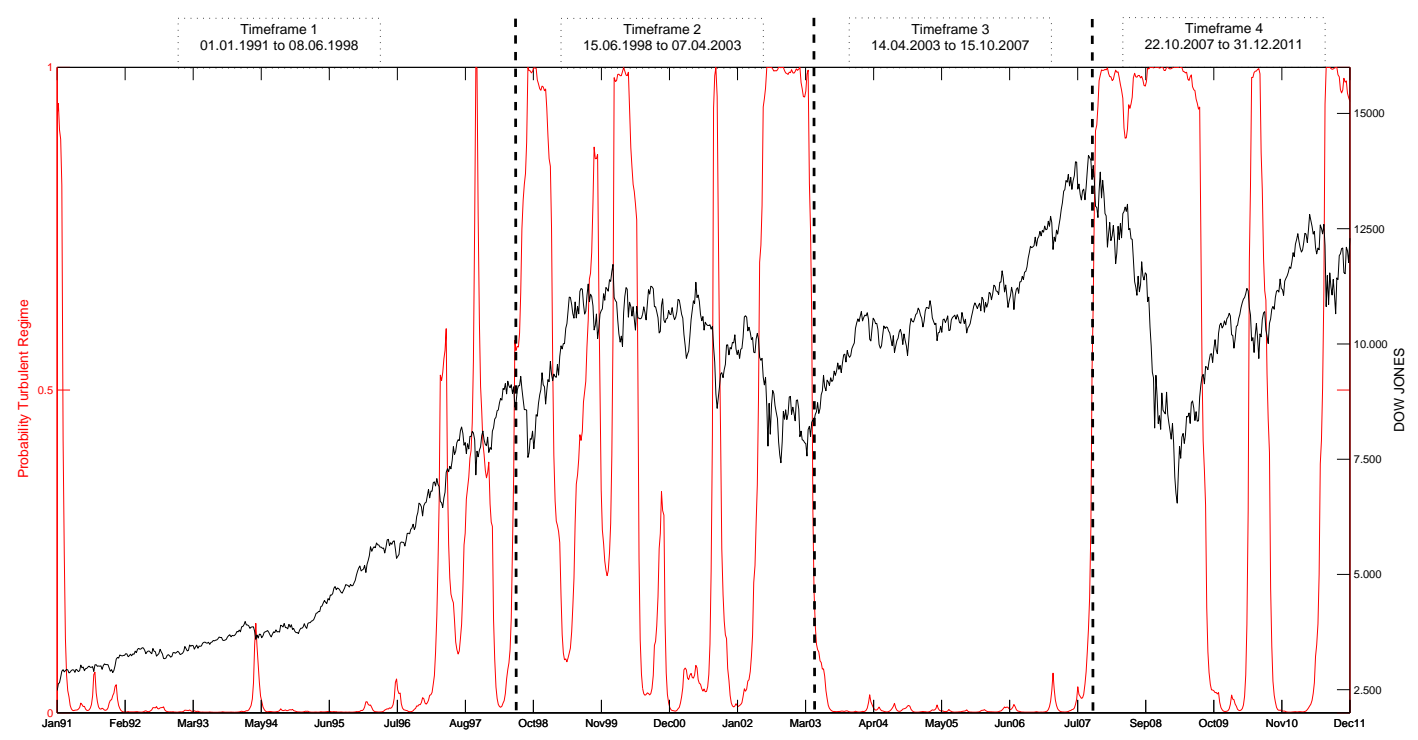

Figure 1: Regime switching model - DOW JONES

the total timeframe into four sub-periods - two normal states (from 01.01.1991 to 08.06.1998 and from 14.04.2003 to 15.10.2007) and two turbulent states (from 15.06.1998 to 07.04.2003 and from 22.10.2007 to 31.12.2011). Hamilton (1989) concludes that his model makes very clear decisions about the probability of being in a certain state. In Hamilton's context this means that only few smoothed probabilities should lie between 0.3 and 0.7 and the algorithm usually identifies fairly clear decisions about the states. With this knowledge he suggests using the decision criterion smoothed probability $\geq 0.5$ in a two state model. In the first sub-period we treat the two short periods with a high smoothed probability as outliers and therefore, consider them to be within a normal time. In the second and fourth sub-period we face times switching from turbulent to normal and back to turbulent with high frequency. We apply Hamilton's (1989) decision criteria and demand an expected smoothed probability $\mu_{s m} \geq 0.5$ for a turbulent time. Therefore, we perform an approximate Gauss test with a $95 \%$ significance level and the null hypothesis $H_{0}: \mu \geq 0.5$ and $H_{1}: \mu<0.5$ as the alternative hypothesis. For more information about the regime

\footnotetext{
${ }^{2}$ As proposed by Alexander (2008) we use the package "MS regress", which was provided by Perlin (2007) for the implementation
} in MATLAB. 
switching model and the applied approximate Gauss-test see appendix A2.

To sum up, we classify our four sub-periods as shown in table 4 and use this division in the subse-

\begin{tabular}{cc}
\hline \hline Timeframe & Regime switching state \\
\hline 01.01 .1991 to 08.06 .1998 & normal \\
15.06 .1998 to 07.04 .2003 & turbulent \\
14.04 .2003 to 15.10 .2007 & normal \\
22.10 .2007 to 31.12 .2011 & turbulent \\
\hline
\end{tabular}

Table 4: Classification sub-periods into normal and turbulent times

quent sections. We also do a robustness test by using the safe haven theory. The results are also shown in appendix A2.

\section{Empirical results}

At first, we try to fit a VG process over the total period of 21 years by means of the six presented estimation methods. Table 5 demonstrates that the quality of the fitting rates (=percentages of shares which follow a VG process) tested by the KS test is not acceptable except for the $\chi^{2}$ method. In contrast to the simulated VG process data, we even notice a strong decrease of the fitting rates with increasing levels of significance. Furthermore, $\mu_{d}$ indicates the mean maximum deviation of the 30 empirical samples. As is the case for the fitting rates, $\mu_{d}$ is too large and therefore does not reveal a high-quality approximation for all methods. Only the $\chi^{2}$ method positively stands out from these methods. For a robustness test of the fitting rates, have a look at the $\chi^{2}$ test's results in appendix A3 (see table 15). These results make

\begin{tabular}{l|cccccc}
\hline \hline & SMOM & MOM & MLE & BI & ECF & $\chi^{2}$ \\
\hline$\mu_{d}$ & 7.96 & 8.37 & 6.21 & 8.75 & 8.79 & 2.43 \\
$1 \%$ & 0 & 0 & 3.33 & 6.67 & 0 & 50 \\
$5 \%$ & 0 & 0 & 0 & 6.67 & 0 & 26.67 \\
$10 \%$ & 0 & 0 & 0 & 3.33 & 0 & 26.67 \\
$15 \%$ & 0 & 0 & 0 & 3.33 & 0 & 20 \\
$20 \%$ & 0 & 0 & 0 & 3.33 & 0 & 20 \\
\hline
\end{tabular}

All fitting rates are indicated in $\%$.

Table 5: KS test: fitting rates of all estimation methods - total timeframe

clear that it is hardly possible to estimate a VG process over a very long period. Problems result from data covering too many different market phases in which the stocks change their behavior. This means that the increments' or the log returns' PDF, resp., has various symmetries, fat tails on the left or right end of the distribution or a time varying volatility as most stocks behave differently during the different periods. Table 10 presents - among other things - the parameter estimation results via the $\chi^{2}$ method and the corresponding significance levels over the period from 1991 to 2011. We observe that it is quite difficult to get significant results for the finance \& insurance sector (for classification of sectors see section 3.2). Taking the descriptive statistics (see table 12 in appendix A1) into account it becomes apparent 
that the finance \& insurance sector has had a very high volatility, which has also led to a high kurtosis, particularly since the beginning of the financial crisis. The VG process tries to cover this stock behavior with $\sigma$ and $\nu$. The comparison of the finance \& insurance sector's $\nu$ with other sectors' features reveals a large upward deviation. It seems that the change of the market behavior of financial institutions' stocks in the last two decades has also affected the fitting characteristics of the stochastic processes and therefore emphasizes our hypotheses $\mathrm{H} 2$ and $\mathrm{H} 3$. In contrast to the highly volatile financial \& insurance sector, the results for the more defensive sub-sectors electrical \& component, trade \& food and oil \& chemistry or the typical cyclical engineering sector are generally more significant. From the results we learn that a randomly selected timeframe used for fitting a stochastic process does not ensure significant results.

With this background knowledge we run the same parameter estimations for the four timeframes identified by the regime switching model and the safe haven theory. Tables 6, 7, 8 and 9 clearly show increasing fitting rates for all estimation methods compared to the total timeframe estimation (see table 5). For a robustness check see tables 16, 17, 18 and 19 in appendix A3. While, for example, the fitting rate for the $\chi^{2}$ method is $50 \%$ ( $1 \%$ significance level) over the total timeframe, the fitting rates vary within the interval $[70 \%, 100 \%]$ over the four identified timeframes. This fact highlights that the data used to fit a model plays an important role and a well-defined period of the data set increases the probability of obtaining high-quality estimations. Besides, we also consider the mean maximum absolute deviation $\mu_{d}$ between the estimated and the empirical CDF. In turbulent timeframes this indicator is smaller (2.45 and 2.01 , resp.) than in normal timeframes ( 3.55 and 2.57, resp.). Further, there is a trend that the deviations themselves decrease in general in normal and turbulent timeframes (as to the $\chi^{2}$ method from 3.55 to 2.01). Again, these trends reaffirm that stock markets are more likely to follow a VG process. We cannot reject our hypothesis $\mathrm{H} 2$ as we obtain strongly improved fitting rates from the application of the regime switching model. We find an outperformance of the $\chi^{2}$ method for all sub-periods and the total period. The MLE method is the second best approach and particularly outperforms the moment based estimation methods in the $\mu_{d}$ figures. The BI method only seems to work well in a highly volatile market phase (timeframe 4) and does not justify the efforts undertaken. These results confirm the simulated VG process' results and our first hypothesis $\mathrm{H} 1$.

\begin{tabular}{l|cccccc}
\hline \hline & SMOM & MOM & MLE & BI & ECF & $\chi^{2}$ \\
\hline$\mu_{d}$ & 5.52 & 5.5 & 4.63 & 13.54 & 5.59 & 3.55 \\
$1 \%$ & 30 & 30 & 30 & 3.33 & 30 & 70 \\
$5 \%$ & 20 & 20 & 20 & 0 & 20 & 53.33 \\
$10 \%$ & 6.67 & 6.67 & 6.67 & 0 & 6.67 & 40 \\
$15 \%$ & 0 & 0 & 0 & 0 & 0 & 26.67 \\
$20 \%$ & 0 & 0 & 0 & 0 & 0 & 16.67 \\
\hline \multicolumn{7}{r}{ All fitting rates are indicated in $\%$}
\end{tabular}

Table 6: KS test: fitting rates of all estimation methods - timeframe 1

So far we have focused on the fitting rates and the quality of the estimations. Now we start a more detailed analysis of the stocks' parameters of the VG process (see table 10). At first, we take the parameters of the total timeframe and the four sub-periods into account. We see that the total timeframe tends to average 


\begin{tabular}{l|cccccc}
\hline \hline & SMOM & MOM & MLE & BI & ECF & $\chi^{2}$ \\
\hline$\mu_{d}$ & 5.17 & 5.14 & 4.06 & 7.93 & 5.26 & 2.45 \\
$1 \%$ & 63.33 & 63.33 & 80 & 3.33 & 63.33 & 90 \\
$5 \%$ & 50 & 50 & 60 & 3.33 & 50 & 90 \\
$10 \%$ & 40 & 40 & 43.33 & 3.33 & 33.33 & 90 \\
$15 \%$ & 30 & 30 & 33.33 & 0 & 26.67 & 90 \\
$20 \%$ & 23.33 & 23.33 & 26.67 & 0 & 23.33 & 90 \\
\hline
\end{tabular}

All fitting rates are indicated in \%.

Table 7: KS test: fitting rates of all estimation methods - timeframe 2

\begin{tabular}{l|cccccc}
\hline \hline \multicolumn{1}{c}{ SMOM } & MOM & MLE & BI & ECF & $\chi^{2}$ \\
\hline$\mu_{d}$ & 7.11 & 6.98 & 4.59 & $40.81^{*}$ & 7.29 & 2.57 \\
$1 \%$ & 53.33 & 53.33 & 56.67 & 6.67 & 53.33 & 90 \\
$5 \%$ & 46.67 & 46.67 & 50 & 6.67 & 46.67 & 90 \\
$10 \%$ & 46.67 & 46.67 & 46.67 & 6.67 & 46.67 & 90 \\
$15 \%$ & 43.33 & 43.33 & 43.33 & 6.67 & 40 & 90 \\
$20 \%$ & 43.33 & 43.33 & 43.33 & 6.67 & 40 & 90
\end{tabular}

* This figure is biased as some shares could not be fitted correctly. All fitting rates are indicated in $\%$.

Table 8: KS test: fitting rates of all estimation methods - timeframe 3

\begin{tabular}{l|cccccc}
\hline \hline \multicolumn{1}{c}{ SMOM } & MOM & MLE & BI & ECF & $\chi^{2}$ \\
\hline$\mu_{d}$ & 7.96 & 7.9 & 6.4 & 3.51 & 8.5 & 2.01 \\
$1 \%$ & 23.33 & 23.33 & 36.67 & 93.33 & 23.33 & 100 \\
$5 \%$ & 20 & 20 & 36.67 & 80 & 16.67 & 100 \\
$10 \%$ & 16.67 & 16.67 & 23.33 & 73.33 & 13.33 & 100 \\
$15 \%$ & 13.33 & 13.33 & 10 & 70 & 10 & 96.67 \\
$20 \%$ & 6.67 & 6.67 & 6.67 & 70 & 3.33 & 93.33 \\
\hline
\end{tabular}

All fitting rates are indicated in $\%$.

Table 9: KS test: fitting rates of all estimation methods - timeframe 4 
over the four sub-timeframes. This means, for example, that we overestimate $\sigma, \theta$ or $\nu$ in a normal period and underestimate them in a turbulent period. With respect to simulation and forecasting applications of asset prices by means of a VG process model this can lead to enormous biases. While $\sigma$ and $\nu$ errors particularly lead to a mismatch of the actual market behavior, a $\theta$ error results in a wrong assumption of the log returns' symmetry. In normal timeframes there is a trend towards a right symmetric stochastic process, whereas in turbulent timeframes there is a trend towards a left symmetric VG process' behavior.

Having looked at the VG process parameters in a more general manner, we continue with some detailed information about the sectors:

Information \& entertainment and electrical \& component:

We notice different behavior in the two turbulent periods. During the dot-com crisis, these two sectors have a higher $\sigma$ and $\nu$ in relation to the other sectors, while these sectors are less volatile during the financial crisis (see table 12 in appendix A1). Particularly, enterprises in the telecommunication and technology sector reveal a stable performance and offer a good opportunity for diversification.

Finance \& insurance:

This sector has an extremely high $\sigma$ and $\nu$ during the financial crisis. The VG process is able to capture this behavior according to the KS test and the respective significance levels. Therefore, the VG process and the jumps generated by a gamma function using the parameter $\nu$ are helpful approaches to cover this difficult market behavior. These findings are in contrast to the first timeframe where the finance $\&$ insurance stocks do not directly follow a VG process. This change in market behavior highlights the transition from a stable to a volatile and nervous sector as a consequence of a financial crisis and political and economic changes.

Engineering:

This sector seems to be strongly dependent on the current economic situation. In turbulent phases $\sigma$ and $\nu$ increase. For that reason, the VG process is a good procedure for modeling stocks of cyclical industries.

Chemistry \& oil and trade \& food:

The VG process parameters $\sigma$ and $\nu$ show that these sectors decouple from the rest of the market in turbulent phases. The parameters indicating nervous markets are lower than those of other sectors. From a risk perspective, we can - again - find some opportunities for diversification.

To sum up, the parameters analysis emphasizes the importance of a clearly defined separation of normal and turbulent market phases and verifies our second hypothesis H2. Additionally, the examination of the four parameters allows a more detailed analysis of stocks from a risk perspective. In some cases, there is only a concentration on the mean and the variance. The VG process allows an extended consideration of risk by also taking the symmetry of stocks' log return PDF, the kurtosis or the market activity with the parameter $\nu$ into account. 


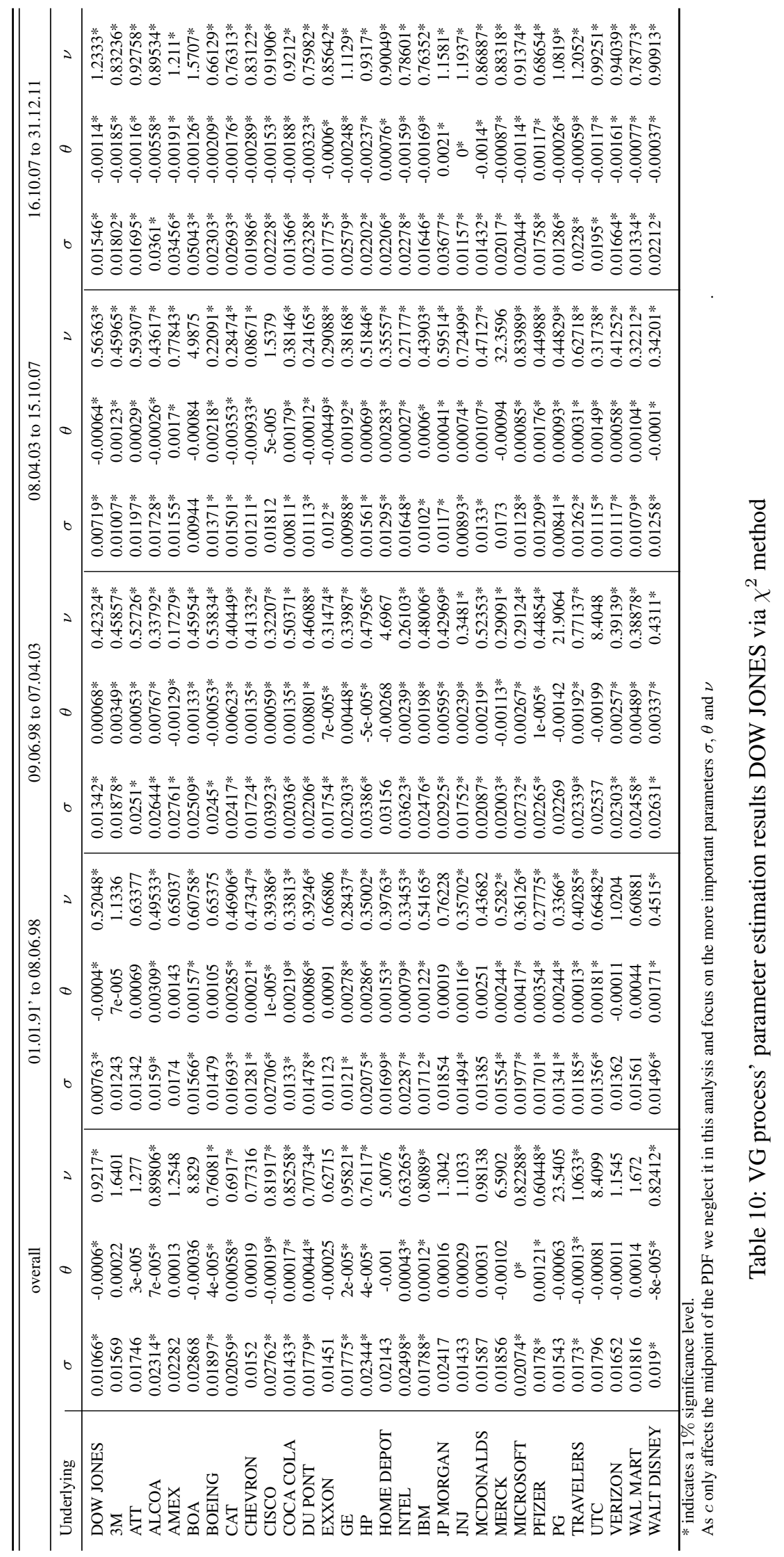




\section{Discussion}

After presenting the empirical results, we put them in relation to each other. The quality of a VG process depends on the estimation method used. Unlike comparable cases, where there often exists a trade off between estimation quality and estimation time, the $\chi^{2}$ method allows a high quality estimation by keeping the estimation effort acceptable. Furthermore, we notice that we even get high fitting rates for the $\chi^{2}$ method when we apply the very restrictive KS test. This fact verifies the good VG process approximation by means of the $\chi^{2}$ method.

We contribute to existing literature, such as Seneta (2004) or Finlay and Seneta (2008), by comparing all estimation methods with a large empirical data set of the DOW JONES for a period of more than 21 years. In contrast to Seneta (2004) and Finlay and Seneta (2008), we do not choose the timeframes for fitting the VG process randomly, we apply a regime switching model for identifying normal and turbulent timeframes. This additional aspect offers a good opportunity for comparing the estimated VG process parameters over time for an underlying. We observe that the parameters vary over time and this modeling approach is a progress in avoiding the over- or underestimation of the actual parameters. However, we are aware that the differentiation between normal and turbulent timeframes is only based on the DOW JONES and not on a single asset decision. Therefore, our results and the behavior of an asset are always compared to the market behavior of the DOW JONES. Nevertheless, the regime switching model increases the fitting rates of the six estimation methods significantly. We also notice that markets are more likely to follow jump processes, such as the VG process, as we identified increasing fitting rates over the four timeframes, particularly for the $\chi^{2}$ method. Besides improved fitting rates, the regime switching model highlights that VG process parameters vary over time and the sensitivity of the VG process itself becomes apparent. As a consequence, we state that a correct estimation method selection is essential for a proper application of the VG process in the context of modeling share returns.

\section{Conclusion}

We have taken the common knowledge about the VG process (see Madan and Seneta (1987), Madan and Seneta (1990), Seneta (2004), Finlay and Seneta (2008), amongst others) and the knowledge about the estimation methods of the VG process estimation methods by means of a simulated VG process data set (see Finlay and Seneta (2008)) and applied it on a large empirical data set, the DOW JONES. Based on the theoretical background we develop three hypotheses, each of which includes an aspect of practical usage of the VG process. We reach our aim to improve the quality of a VG process by integrating a regime switching model developed by Hamilton (1989) and Hamilton (2005) into the VG process framework, and in this way, identify VG process parameters which depend on timeframes (hypothesis H2). This approach results in the improvement of fitting rates (hypothesis H3), which are determined by the conservatively adjusted KS test for CDF. Finlay and Seneta (2008) suggest using a MLE method or $\chi^{2}$ method in order to fit a VG process by means of a simulated data set. We can verify this knowledge for empirical data, but in contrast to Finlay and Seneta (2008), we find a superiority of the $\chi^{2}$ method for empirical data (hypothesis H1).

The regime switching model allows us to have a closer look at the parameters of the VG process over the various normal and turbulent regimes over our daily data set comprising 21 years. Furthermore, we can learn that such a differentiation helps to avoid an over- or underestimation of the real VG process stock market behavior. 
However, we state that our results only cover the DOW JONES and the new knowledge about the VG process is limited to this data set. The scope of the analysis could be broadened to cover other markets such as the commodities, currency or bond markets by extending the data set. Furthermore, this paper focuses on the identification of the estimation method fitting the VG process best. On the basis of these results we could compare the VG process with other stochastic models.

To sum up, our findings should ensure a better understanding of the VG process in connection with an empirical dataset. This empirical study on the VG process should provide a useful basis for further research on the permanent consideration of the combination of the regime switching model and the VG process. 


\section{References}

Aldrich, J., 1997. R. A. Fisher and the making of maximum likelihood 1912-1922. Statistical Science 3, $162-176$.

Alexander, C., 2008. Market risk analysis - practical financial econometrics Vol. 2, first ed., The Wiley Finance Series, New Jersey.

Berkson, J., 1980. Minimum chi-square, not maximum likelihood. The Annals of Statistics 8, 457-487.

Black, F., Scholes, M., 1973. The pricing of options and corporate liabilities. Journal of Political Economy 81, 637-654.

Cerra, V., Saxena, S. W., 2003. Did output recover from the Asian crisis, International Monetary Fund, Working Paper.

Cont, R., Tankov, P., 2004. Financial modelling with jump processes, first ed. CRC Press, New York.

Cox, J. C., Ross, S. A., 1976. The valuation of options for alternative stochastic processes. Journal of Financial Economics 3, 145-166.

Daal, E. A., Madan, D. B., 2005. An empirical examination of the variance gamma model for foreign currency options. The Journal of Business 78, 2121-2152.

Efron, B., 1982. The 1981 Wald memorial lectures - maximum likelihood and decision theory. The Annals of Statistics 10, 340-356.

Fama, E. F., 1965. The behavior of stock-market prices. The Journal of Business 38, 34-105.

Finlay, R., Seneta, E., 2006. Stationary-increment student and variance-gamma processes. Journal of Applied Probability 43, 441-453.

Finlay, R., Seneta, E., 2008. Stationary-increment variance-gamma and t models - simulation and parameter estimation. International Statistical Review 76, 167-186.

Hamilton, J. D., 1988. Rational expectations econometric analysis of changes in regime - an investigation of the term structure of interest rates. Journal of Economic Dynamics and Control 12, 385-423.

Hamilton, J. D., 1989. A new approach to the economic analysis of nonstationary time series and the business cycle. Econometrica 57, 357-384.

Hamilton, J. D., 1994. Time series analysis, first ed. Princeton University Press, New Jersey.

Hamilton, J. D., 2005. Regime switching models, first ed. Palgrave Dictionary of Economics, New Jersey.

Heston, S. L., 1993a. A closed-form solution for options with stochastic volatility with applications to bond and currency options. The Review of Financial Studies 6, 327-343.

Heston, S. L., 1993b. Invisible parameters in options prices. The Journal of Finance 48, 933-947.

Hsu, D. A., Miller, R. B., Winchern, D. W., 1974. On the stable paretian behavior of stock-market prices. Journal of the American Statistical Association 69, 108-113. 
Hull, J., White, A., 1987. The pricing of options on assets with stochastic volatility. The Journal of Finance 42, 281-300.

Jeanne, O., Masson, P., 2000. Currency crises, sunspots and markov-switching regimes. Journal of International Economics 50, 327-350.

Jones, E. P., 1984. Option arbitrage and strategy with large price changes. Journal of Financial Economics $13,91-113$.

Luciano, E., Schoutens, W., 2006. A multivariate jump-driven financial asset model. Quantitative Finance $6,385-402$.

Madan, D. B., Seneta, E., 1987. Simulation of estimates using the empirical characteristic function. International Statistical Review 55, 153-161.

Madan, D. B., Seneta, E., 1990. The variance gamma VG model for share market returns. The Journal of Business 63, 511-524.

Madan, D. P., Carr, P. P., Chang, E. C., 1998. The variance gamma process and option pricing. European Finance Review 2, 79-105.

Mandelbrot, B., 1963. The variation of certain speculative prices. The Journal of Business 36, 394-419.

Massey, F. J., 1951. The Kolmogorov-Smirnov test for goodness of fit. Journal of the American Statistical Association 46, 68-78.

Merton, R. C., 1976. Option pricing when underlying stock returns are discontinuous. Journal of Financial Economics 3, 125-144.

Officer, R. R., 1972. The distribution of stock returns. Journal of the American Statistical Association $67,807-812$.

Osborne, M. F. M., 1959. Brownian motion in the stock market. Operations Research 7, 145-173.

Perlin, M., 2007. MS regress - the MATLAB package for Markov regime switching models, Working Paper.

Praetz, P. D., 1972. The distribution of share price changes. The Journal of Business 45, 49-55.

Roberts, G., Rosenthal, J., 2004. General state space markov chains and MCMC algorithms. Probability Surveys 1, 20-71.

Roberts, H. V., 1965. Probabilistic prediction. Journal of the American Statistical Association 60, 50-62.

Schoutens, W., 2003. Levy processes in finance: pricing financial derivatives, first ed., The Wiley Series in Probability and Statistics, New Jersey.

Seneta, E., 2004. Fitting the Variance Gamma model to financial data. Journal of Applied Probability 41, 177-187.

Seneta, E., Tjetjep, A., 2006. Skewed normal-variance-mean models for asset pricing and the method of moments. International Statistical Review 74, 109-126.

Yu, J., 2004. Empirical characteristic function estimation and its applications. Econometric Reviews 23, 93-123. 


\section{Appendix A1}

There are various ways to measure the fitting quality between the real model and the estimated model. A common approach is to compare the estimated PDF or the cumulative distribution function (CDF) with the respective empirical PDF or CDF. Literature mainly proposes the $\chi^{2}$ test, which is very similar to the $\chi^{2}$ method. The close connection to the $\chi^{2}$ method can lead to less objectivity and result in a bias. Bearing our aim of identifying the best fitting method in mind, we choose the KolmogorovSmirnov (KS) test as suggested by Massey (1951). This test is based on the maximum difference between an empirical and a hypothetical CDF. For the reasons mentioned, we first run a KS test, and then back-test the results with the $\chi^{2}$ test. The KS test assumes that the log returns follow a VG process as null hypothesis. The alternative hypothesis simply rejects this assumption. The main framework consists of the comparison between the maximum absolute deviation of the estimated CDF and empirical $\mathrm{CDF}, F_{X}^{E}\left(x_{i}\right)=\frac{1}{N} \sum_{j=1}^{N} 1_{\left\{x_{j} \leq x_{i}\right\}}$, where $X_{i}=\left(X_{1}, \ldots, X_{N}\right)$ are the $N$ observed log returns of the VG process and $1_{\left\{x_{j} \leq x_{i}\right\}}$ is 1 if $x_{j} \leq x_{i}$, else 0 . By means of the estimated parameter set $\eta$ and the PDF of the VG process (see formula 4), the estimated CDF of the VG process $F_{X}(x)$ is defined as $F_{X, \eta}\left(x_{i}\right)=\int_{-\infty}^{x_{i}} f_{X, \eta}(x) d x$. In the last step, the maximum absolute deviation $d$ between $F_{X}^{E}\left(x_{i}\right)$ and $F_{X}\left(x_{i}\right), d=\max _{i=1 \ldots N}\left|F_{X}^{E}\left(x_{i}\right)-F_{X, \eta}\left(x_{i}\right)\right|$, is calculated and compared with the critical values (see table 11) as defined by Massey (1951). $\alpha$ stands for the significance level. If $d$ exceeds $d_{\alpha}$, we reject the null hypothesis. The KS test is sometimes criticized as the decision is based on one maximum deviation

\begin{tabular}{|c|ccccc|}
\hline \hline$\alpha$ & $1 \%$ & $5 \%$ & $10 \%$ & $15 \%$ & $20 \%$ \\
\hline$d_{\alpha}$ & $1.63 \sqrt{N}$ & $1.36 \sqrt{N}$ & $1.22 \sqrt{N}$ & $1.14 \sqrt{N}$ & $1.07 \sqrt{N}$ \\
\hline \hline
\end{tabular}

Table 11: Significance levels KS test

only. For that reason, an estimated CDF that fits badly to the empirical CDF at one point in time only is regarded as non-convenient, although the other points in time are estimated correctly. We treat this aspect by taking it as a more conservative method.

The $\chi^{2}$ test is similar to the $\chi^{2}$ method. It is a common approach for testing the quality of an estimated CDF. Let $\eta, r, B, O_{i}$ and $\tilde{O}_{i}$ be defined as described in the section dealing with the $\chi^{2}$ method part (see section 2) and $Q$ be defined as the intervals. By means of these parameters, the $\chi^{2}$ test value $\chi^{2}(\eta)=\sum_{i=1}^{Q} \frac{\left(O_{i}-\tilde{O}_{i}(\eta)\right)^{2}}{\tilde{O}_{i}(\eta)}$ is calculated. Subsequently, $\chi^{2}(\eta)$ is compared to the quantile $(1-\alpha)$ of the $\chi^{2}$ distribution with $Q-5$ degrees of freedom for various significance levels $\alpha$ according to Finlay and Seneta (2008). If $\chi^{2}(\eta)$ exceeds the quantile, we reject the null hypothesis that the observations $X_{t}$ follow a VG process. The degrees of freedom are calculated as the number of intervals minus the number of estimated parameters minus one. Instead of using $I$ intervals, as in the $\chi^{2}$ method, we apply $Q$ ( $Q=10$ which is similar to $10 \%$ quantile bands). We change this technical aspect in order to reduce the advantage of the $\chi^{2}$ method over the other estimation methods and, in this case, differ from Finlay and Seneta (2008). They apply wider intervals on the right and left hand side of the distribution and smaller intervals in between. 


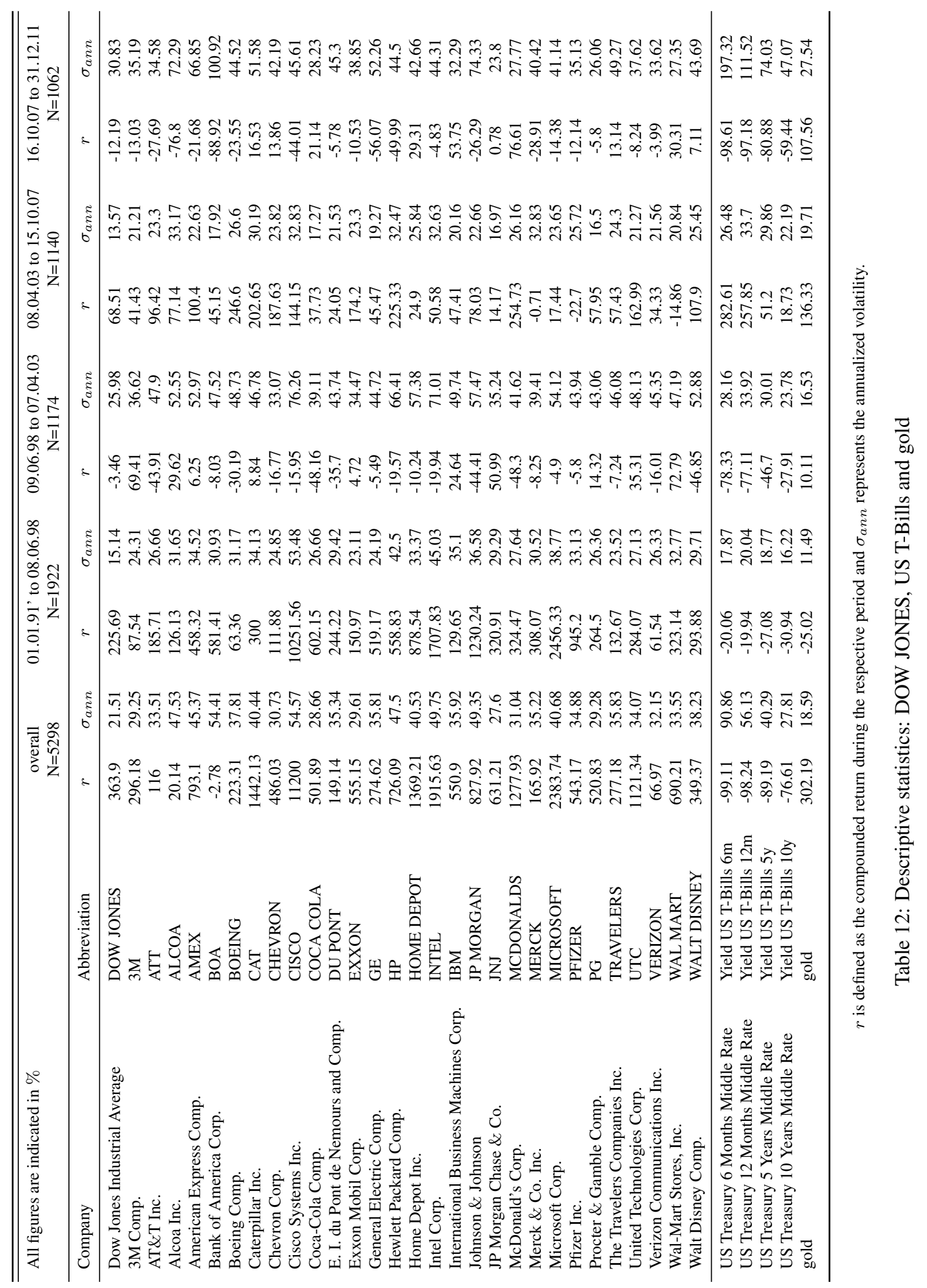




\section{Appendix A2}

As we have no idea about the distribution of the smoothed probabilities, we do not assume any distribution for calculating the test statistic value $V$. Table 13 demonstrates that we can reject $H_{o}$ in the first and third sub-period, but not in other periods.

\begin{tabular}{cccccc}
\hline \hline Timeframe & Observations & $\mu_{s m}$ & $\sigma_{s m}$ & $\mathrm{~V}$ & Rejection interval \\
\hline 01.01 .1991 to 08.06 .1998 & 387 & 0.067651 & 0.16408 & -51.8376 & $(-\infty,-1.6449)$ \\
15.06 .1998 to 07.04 .2003 & 252 & 0.48732 & 0.40017 & -0.50313 & $(-\infty,-1.6449)$ \\
14.04 .2003 to 15.10 .2007 & 236 & 0.013478 & 0.036818 & -202.9983 & $(-\infty,-1.6449)$ \\
22.10 .2007 to 31.12 .2011 & 219 & 0.60208 & 0.44601 & 3.3869 & $(-\infty,-1.6449)$ \\
\hline
\end{tabular}

Table 13: Descriptive statistics: approximate Gauss test for smoothed probabilities

We also present the estimated transition matrix $P^{*}$ for the regime switching model for the DOW JONES

$$
P^{*}=\left(\begin{array}{cc}
0.99 & 0.01 \\
\left(0.00^{* * *}\right) & \left(0.01^{* * *}\right) \\
0.04 & 0.96 \\
\left(0.00^{* * *}\right) & \left(0.00^{* * *}\right)
\end{array}\right),
$$

which provides the probabilities of switching from one state to another. The figures in brackets denoted are the test values and the significances levels, with ${ }^{* * *}$ indicating a $1 \%$ significance level.

For running a robustness test which is in accordance with the safe haven theory, we have a look at the correlations between the DOW JONES, US T-Bills yields and gold. In turbulent times, the correlation between the DOW JONES and the US T-Bills yields increases. This means that while stock markets decrease, US T-Bills prices increase (yields =decrease). Furthermore, gold decouples from the DOW JONES in turbulent times.

\begin{tabular}{cccccc}
\hline \hline Timeframe & $\begin{array}{c}\text { Yield US } \\
\text { T-Bills 6m }\end{array}$ & $\begin{array}{c}\text { Yield US } \\
\text { T-Bills 12m }\end{array}$ & $\begin{array}{c}\text { Yield US } \\
\text { T-Bills 5y }\end{array}$ & $\begin{array}{c}\text { Yield US } \\
\text { T-Bills 10y }\end{array}$ & gold \\
\hline 01.01.1991 to 08.06.1998 & 0.32654 & 0.29208 & -0.25329 & -0.51722 & -0.45986 \\
15.06.1998 to 07.04.2003 & 0.59068 & 0.61947 & 0.76689 & 0.80246 & -0.67852 \\
14.04 .2003 to 15.10.2007 & 0.761 & 0.75848 & 0.73629 & 0.63986 & 0.90282 \\
22.10 .2007 to 31.12.2011 & 0.45577 & 0.41158 & 0.24329 & 0.23187 & 0.31944 \\
\hline
\end{tabular}

Table 14: Correlation between DOW JONES and US T-Bills and gold 


\section{Appendix A3}

The following six tables show the fitting rates based on the $\chi^{2}$ test. As mentioned above we use these results as a robustness check for the KS test. Each time a robustness test is needed, a reference can be found in the main part of this paper. The results of the $\chi^{2}$ test are in accordance with the KS test and verify the $\chi^{2}$ method as the best estimation method of the VG process. All in all, the results only differ in that they reveal lower fitting rates.

\begin{tabular}{l|cccccc}
\hline \hline \multicolumn{1}{l|}{ SMOM } & MOM & MLE & BI & ECF & $\chi^{2}$ \\
\hline $1 \%$ & 0 & 0 & 0 & 0 & 0 & 6.67 \\
$5 \%$ & 0 & 0 & 0 & 0 & 0 & 3.33 \\
$10 \%$ & 0 & 0 & 0 & 0 & 0 & 0 \\
$15 \%$ & 0 & 0 & 0 & 0 & 0 & 0 \\
$20 \%$ & 0 & 0 & 0 & 0 & 0 & 0 \\
\hline
\end{tabular}

All fitting rates are indicated in $\%$.

Table 15: $\chi^{2}$ test: fitting rates of all estimation methods - total timeframe

\begin{tabular}{l|cccccc}
\hline \hline \multicolumn{1}{l}{ SMOM } & MOM & MLE & BI & ECF & $\chi^{2}$ \\
\hline $1 \%$ & 16.67 & 16.67 & 16.67 & 0 & 13.33 & 33.33 \\
$5 \%$ & 3.33 & 3.33 & 3.33 & 0 & 3.33 & 13.33 \\
$10 \%$ & 0 & 0 & 0 & 0 & 0 & 10 \\
$15 \%$ & 0 & 0 & 0 & 0 & 0 & 10 \\
$20 \%$ & 0 & 0 & 0 & 0 & 0 & 6.67 \\
\hline
\end{tabular}

All fitting rates are indicated in $\%$.

Table 16: $\chi^{2}$ test: fitting rates of all estimation methods - timeframe 1 


\begin{tabular}{l|cccccc}
\hline \hline & SMOM & MOM & MLE & BI & ECF & $\chi^{2}$ \\
\hline $1 \%$ & 16.67 & 20 & 16.67 & 0 & 16.67 & 86.67 \\
$5 \%$ & 13.33 & 13.33 & 13.33 & 0 & 13.33 & 66.67 \\
$10 \%$ & 6.67 & 6.67 & 6.67 & 0 & 6.67 & 56.67 \\
$15 \%$ & 6.67 & 6.67 & 6.67 & 0 & 6.67 & 40 \\
$20 \%$ & 6.67 & 6.67 & 6.67 & 0 & 6.67 & 40 \\
\hline
\end{tabular}

All fitting rates are indicated in $\%$.

Table 17: $\chi^{2}$ test: fitting rates of all estimation methods - timeframe 2

\begin{tabular}{l|cccccc}
\hline & SMOM & MOM & MLE & BI & ECF & $\chi^{2}$ \\
\hline $1 \%$ & 43.33 & 40 & 43.33 & 13.33 & 40 & 86.67 \\
$5 \%$ & 30 & 26.67 & 30 & 13.33 & 26.67 & 76.67 \\
$10 \%$ & 26.67 & 23.33 & 26.67 & 10 & 23.33 & 63.33 \\
$15 \%$ & 23.33 & 20 & 23.33 & 10 & 23.33 & 56.67 \\
$20 \%$ & 20 & 16.67 & 20 & 10 & 20 & 43.33 \\
\hline
\end{tabular}

All fitting rates are indicated in $\%$.

Table 18: $\chi^{2}$ test: fitting rates of all estimation methods - timeframe 3

\begin{tabular}{l|cccccc}
\hline \hline & SMOM & MOM & MLE & BI & ECF & $\chi^{2}$ \\
\hline $1 \%$ & 6.67 & 6.67 & 10 & 46.67 & 6.67 & 90 \\
$5 \%$ & 3.33 & 3.33 & 3.33 & 26.67 & 3.33 & 83.33 \\
$10 \%$ & 3.33 & 3.33 & 3.33 & 20 & 3.33 & 70 \\
$15 \%$ & 3.33 & 3.33 & 3.33 & 16.67 & 3.33 & 53.33 \\
$20 \%$ & 0 & 0 & 0 & 16.67 & 0 & 43.33 \\
\hline \multicolumn{7}{c}{ All fitting rates are presented in \%. }
\end{tabular}

Table 19: $\chi^{2}$ test: fitting rates of all estimation methods - timeframe 4 